Biological Psychology 10 (1980) 7-19

(c) North-Holland Publishing Company

\title{
THE INFLUENCE OF TASK RELEVANCE AND STIMULUS INFORMATION ON HABITUATION OF THE VISUAL AND THE SKIN CONDUCTANCE ORIENTING REACTION
}

\author{
M.N. VERBATEN, J.C. WOESTENBURG and W. SJOUW \\ Department of Psychophysiology, University of Utrecht, Utrecht, The Netherlands
}

Accepted for publication 7 February 1980

This study investigated the hypothesis that task-relevant stimuli induce orienting reactions (ORs) that are stronger and more resistant to habituation when their information content is high than when it is low. Task-relevance was given to the stimuli by rewarding the subjects for correct recognition at the end of the experiment. The dependent variables in this study were the visual orienting reaction (VOR), the skin conductance reaction (SCR), their habituation scores and the number of spontaneous fluctuations in skin conductance (SFs). 28 subjects received two blocks of 14 trials. Half the subjects received the higher information condition first and then the lower information condition, while the other half received the reversed order. The VOR habituated quickly and was not significantly influenced by information value. SCR amplitudes were larger and SCR-habituation slower to stimuli containing more information. The results were interpreted as supporting the hypothesis that the SCR is associated with a secondary phase of the orienting process.

\section{Introduction}

According to Sokolov (1963) task relevant stimuli do not lose their significance for the subject and thus the orienting reaction (OR) induced by these stimuli will habituate relatively slowly. He demonstrated this by showing that the skin conductance reaction (SCR) evoked by an auditory stimulus did not habituate if this stimulus required a motor reaction.

But not only tasks requiring a motor reaction succeed in delaying habituation of the SCR. Cook and Harris (1937), Grings and Lockhart (1963) and Carroll and Pokora (1976) demonstrated that responses to stimuli signaling an oncoming aversive electrical shock habituated slower compared with control conditions.

Stimuli containing more information should also induce slower habituation of the OR. It seems, however, that this effect can only be obtained when the information containing stimuli have significance for the subject. Harding and Punzo (1971) did not find a difference in SCR amplitudes between stimuli differing in information content (in terms of uncertainty) when they instructed their subjects to attend to these stimuli, but requiring a motor reaction to these stimuli lead to the 
expected difference in SCR amplitudes. Berlyne, Craw, Salapatek and Lewis (1963) found that more complex or incongruous stimuli, containing more information, evoked more SCR's than stimuli containing less information, but only when they instructed their subjects to attend to the stimuli because after the experiment their knowledge of the stimuli would be tested in a recognition test. Spinks and Siddle (1976) found significantly slower SCR habituation to task-relevant visual stimuli with more information.

So, as far as the information value of visual stimuli and SCR habituation are concerned, the task-relevance of the stimuli seems a crucial variable, and in accordance with that conclusion the present authors (Verbaten, Woestenburg and Sjouw, 1979) did not find a significant effect of stimulus information on SCR habituation under non-task relevance conditions. However, in the latter study the visual orienting reaction (VOR), a measure of the direction of the gaze, was also measured and this response habituated more slowly to stimuli containing more information than to those containing less.

In this study we have investigated this problem further by attaching task relevance to stimuli differing in information value. Because the figures constructed by Spinks and Siddle (1976) were employed, we expected to replicate their results. In accordance with our previous results, we also predicted that VOR habituation would be slower in the high information condition.

A second problem investigated in this study was whether the slower SCR habituation to task-relevant stimuli containing more information is part of a more general autonomic arousal increase, or is more specifically related to the processing of these stimuli. Bernstein (1973) showed that giving task relevance to stimuli not only induced more SCRs, but also produced an increase in the number of spontaneous fluctuations in skin conductance (SFs). Number of SFs is often interpreted as indicating non-specific autonomic arousal and appears to correlate highly with SCR habituation (Martin and Rust, 1976). Thus, it might be that the slower habituation of the SCR found by Spinks and Siddle (1976) during the high information condition was caused by a rise in non-specific autonomic arousal during the condition. The alternative possibility is that the slower habituation of the SCR was somehow specifically related to the processing of the task relevant stimuli. In order to investigate this problem we also measured SFs in this experiment.

The VOR is a comparatively infrequently used OR index. The approach to the VOR in this study is identical to that in an earlier study (Verbaten et al., 1979) and is discussed there in some detail.

Summarizing, two hypotheses were investigated in this study. The first was whether task-relevant stimuli differing in information value induced differences in amplitudes and habituation rates of two OR indices, the SCR and the VOR. The second hypothesis was whether the predicted slower habituation of the SCR in the high information condition concurred with a higher frequency of SFs during that condition. 


\section{Method}

\subsection{Subjects}

Subjects were 28 undergraduate students of psychology at the University of Utrecht. There were 12 males and 16 females. Mean age was 22.2 years, (s.d. $=3.9$ years). Subjects wearing glasses kept them on during the experiment.

\subsection{Apparatus}

\subsubsection{Electrooculography (EOG)}

Standard $\mathrm{Ag}-\mathrm{AgCl}$ electrodes in plastic cups were used for the EOG. The electrodes were placed at the outer cathus of each eye for the horizontal EOG. Infraorbital and supra-orbital electrodes were placed in line with the pupil of the left eye for the vertical EOG. Hellige EEG paste was used, because of the low polarization of the electrode paste in combination with the $\mathrm{Ag}-\mathrm{AgCl}$ electrodes. Resistance of the electrodes was never higher than $3 \mathrm{k} \Omega$.

The signals from the electrodes were amplified and filtered by an Elema universal filter (RC time was $36 \mathrm{sec}$, low pass frequency was $15 \mathrm{~Hz}$ ). The long RC time was chosen to cut off slow changes caused by (the small) polarization effects of the electrode-electrolyte combination. Amplifier output was first sent through a $45 \mathrm{~Hz}$ low pass filter to cut off spurious signals and then sent to analogue inputs of the PDP $11 / 40$ computer for on line analogue-digital conversion and scoring.

The sampling rate was $40 \mathrm{~Hz}$. Sampling during calibration started $240 \mathrm{msec}$ after stimulus onset and lasted $200 \mathrm{msec}$. Sampling during the habituation trials and durirg the check procedure started at stimulus onset and lasted 2 sec.

\subsubsection{SCR}

The SCR was measured by a constant voltage system $(0.5 \mathrm{~V})$. Two $\mathrm{AgAg}-\mathrm{Cl}$ pellet electrodes were attached to the palm of the right hand. The electrode paste contained a 0.05 molar concentration of $\mathrm{NaCl}$ in an agar-agar base.

Conductance values were transformed into digital values and then sent to the digital input of the PDP 11/40. Sample frequency of these digital values was $5 \mathrm{~Hz}$. Sampling lasted from 0 till $12 \mathrm{sec}$ after stimulus onset. Scoring was done by the PDP $11 / 40$.

\subsubsection{Stimuli}

The information value of the stimuli used in this experiment (resp. 12 bits and 60 bits) was determined by Spinks and Siddle (1976), who used a procedure developed by Attneave (1954). Although differing in complexity, the stimuli had equal areas of black and white. Build up time of the stimuli on the television monitor was $20 \mathrm{msec}$ and was independent of stimulus complexity. 


\subsubsection{Stimulus conditions}

During the experiment subjects lay on an examination table in an acoustically and electrically shielded room. The upper portion of the examination table was adjustable, so that the subject's head could be adjusted to a position roughly parallel to a television monitor (black and white, 26 inch screen), which was positioned above and in front of the subject, at a distance of $60 \mathrm{~cm}$ from the subject's eyes. Stimuli and instructions were presented to the subjects on this television monitor. Clamps were used to position subject's head so that the centre of the television screen was in the centre of the visual field. Eye movements from the centre of the television screen to any of the four corners of the screen were 28 degrees of arc.

Stimuli presented during the calibration period were the letters $\mathrm{X}$ in the four corners of the screen and the letter $O$ in the centre of the screen. During calibration the duration of these stimuli was $1.3 \mathrm{sec}$ and the intervals between presentations were $3 \mathrm{sec}$. During habituation trials the stimuli containing the two information values were presented in the upper right corner (see fig. 1) for a period of $2.0 \mathrm{sec}$.

The length of intervals between stimulus presentations during habituation trials were randomly chosen and varied between $15 \mathrm{sec}$ and $25 \mathrm{sec}$.

$$
\begin{aligned}
& 220 \\
& 200 \\
& 180 \\
& 160 \\
& 140^{\circ} \\
& 120^{\circ} \\
& 100
\end{aligned}
$$$$
240 X^{1}
$$$$
\begin{aligned}
& 80 . \\
& 60 . \\
& 40 . \\
& 20 .
\end{aligned}
$$

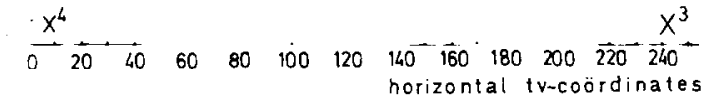

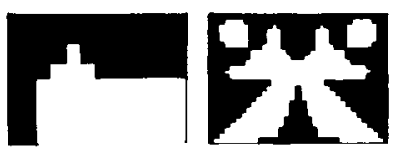

60 bits

Fig. 1. Distances of the four calibration stimuli (X1, X2, X3 and X4) and the information containing stimuli in television-co-ordinates $(256 \times 256)$. The discontinuous lines indicate the two windows, field 1 and field 2 around the information containing stimuli, which were presented during the habituation trials. Horizontal distance is 45 degrees of arc, vertical distance is 36 degrees of arc. 
Eye-movements and SCRs as well as marks of stimulus onset and offset were displayed as ink records on a Mingograph 80 polygraph. Horizontal and vertical EOGs were displayed on a $\mathrm{X}-\mathrm{Y}$ oscilloscope for purposes of checking.

Presentation of stimuli and of instructions and sampling procedures were controlled by the PDP $11 / 40$.

\subsection{Procedure}

On arrival the subjects were familiarized with the procedure and put at ease regarding the methods employed and their part in the experiment. After the electrodes were attached in the instrument room, which adjoined the experimental room, the subject lay down on the examination table and the instruments were connected. The head was fixed in the desired position and the subject was informed that instructions would follow on the television-screen.

The experiment started with a 5 min adaptation period, after which the instructions were presented. These included a request to move as little as possible and not to fall asleep. Eye movement calibration then commenced.

\subsubsection{Calibration}

First the letter $\mathrm{O}$ was presented in the centre of the screen. Then at regular intervals the letter $O$ was followed by the letter $X$ in each of the four corners of the screen. The subject was asked to fixate the letters as accurately as possible. The first trial was used to familiarize the subjects with the calibration procedure. The second time EOGs were sampled and the computer was programmed to construct a twodimensional representation of the four different positions of the letters $X$ as perceived by the subject.

In order to measure the accuracy of the eye movements towards the stimuli during the habituation trials, the computer was programmed to construct two circles (windows) around the stimulus that was to be presented in the upper right corner. The inner circle (field 1) scored maximal accuracy of the eye movements towards these stimuli and the outer circle (field 2) scored less accurate eye movements in the direction of the stimuli (see also section 2.4).

\subsubsection{Habituation}

After the calibration trials the subject was told that a number of figures would be presented on the television screen. Subjects were instructed to pay attention to these figures because at the end of the experiment there would be a recognition test. They were told that money could be earned, but only in the case of two correct recognitions. Then each subject received one block of 14 trials in which a 12-bits figure was presented and one block of 14 trials in which a 60-bits figure was presented. Between the 2 blocks there was a 2 min rest period.

Half the subjects received the block with the 12-bits figure first and then the block with the 60-bits figure. The other half received the reversed order. All figures were presented in the upper right corner of the television-screen. 


\subsubsection{Check}

At the end of each block there was a checking procedure. The subjects were told that a different figure (on precisely the same television co-ordinates as the habituation stimuli) would be presented. They were asked to fixate this new figure accurately. These fixations were scored with the same program as was used for scoring the VORs. This check procedure made it possible to test whether the changes in eye movement behavior seen during the habituation trials were real or determined by artefacts like head movements or drift.

\subsubsection{Recognition test}

At the end of the experiment the subjects were given two multiple choice recognition tests. Subjects had to identify the 12 bits figure and the 60 bits figure between three slightly different ones each.

\subsection{Scoring}

\subsubsection{SCR}

Each change in conductance, larger than $0.01 \mu$ mho, occurring within 0.6 to $6 \mathrm{sec}$ after stimulus onset was scored as an SCR. Amplitude measures were subjected to a square root transformation prior to analysis. Scoring was done on line with a computer program. Reactions lasting shorter than $600 \mathrm{msec}$, from beginning to peak, were not scored as an SCR. Habituation scores were the number of SCRs occurring until the habituation criterion of three consecutive responses of less than $0.01 \mu$ mho was reached.

\subsection{2. $S F$}

A conductance change larger than $0.01 \mu$ mho occurring from 6.0 to $12.0 \mathrm{sec}$ after stimulus onset was scored as an SF. The SF score was the number of SFs occurring in that period.

\subsection{3. $V O R$}

Fixation criterion was $100 \mathrm{msec}$. The VOR was the sum of the fixation times inside field 1 during the period of stimulus presentation.

As can be seen in fig. 1, two fields (field 1 and field 2) were constructed. In an earlier study (Verbaten et a1., 1979) we found that fixations in field 2 did not habituate and were not influenced by the information value of the stimuli, while fixations inside field 1 showed both effects. For this reason fixations inside field 2 were not regarded as part of a VOR.

Measuring the fixations inside field 2 enabled us to test whether the decline of total fixation time inside field 1 was replaced by an increase in fixation time in field 2 . In that case the criterion for a visual orienting reaction could have been too narrow. Changes in field 2 would indicate that such eye movements were influenced by the conditions of this experiment, and thus should be regarded as a part of a VOR. 
The VOR habituation score was the number of VORs until the habituation criterion of three consecutive zero responses was reached.

\section{Results}

The repeated measurements are treated in this study as multiple intercorrelated responses of the same subject. A multivariate analysis of variance (Finn, 1976) was done with order $(O)$ of presentation of information conditions as a between-subjects factor and trials (T) and information (I) as within-subjects factors.

As there were specific expectations in regard to the effect of trials (decrease of response amplitude over trials) and information (slower habituation to the figures with more information) on the SCR and the VOR a 5\% one-tailed level of significance was set in these cases. In the other cases a 5\% two-tailed level of significance was accepted. The dependent variables in this analysis were the VOR, summated fixation times in field 2, SCR amplitudes and SFs. The results of the analysis are summarized in table 1 (see also fig. 2).

The main effect of the factor information was only significant for SCR ampltude. The main effect of the factor trials was significant for the VOR and SCR. It should be noted that neither information nor trials had a significant effect on SFs. Trials $X$ information interaction was not significant. This meant that the differences in SCR amplitudes between trials were equal for the two information groups. Although the effect of trials on field 2 was significant at a $5 \%$ one-tailed level, it was quire small compared to the robust effect of trials on the VOR (fixation time inside field 1). Some small overlap between field 1 and field 2 was indicated by this effect, but in our opinion no revision of our original definition of the VOR was necessary (see also figs. 2(a) and 2(b)).

Table 1

Summary of the multivariate analysis; univariate $F_{S}$ and $d f s$ for the four dependent variables VOR, field 2, SCR and SF's

$\begin{array}{lcclcl}\text { Source } & \text { df } & \text { VOR } & \text { Field 2 } & \text { SCR } & \text { SFs } \\ \text { Order }(O) & & & & & \\ \text { Information }(I) & 1,26 & 0.77 & 0.57 & 0.53 & 1.46 \\ \text { Trials }(T) & 1,26 & 0.55 & 0.03 & 5.60 \mathrm{~b}) & 0.17 \\ O \times I & 1,26 & 36.63^{\mathrm{c})} & 4.89^{\mathrm{a})} & 34.87^{\mathrm{c})} & 0.11 \\ O \times T & 1,26 & 0.02 & 2.06 & 2.92 & 0.32 \\ I \times T & 1,26 & 0.35 & 0.04 & 0.51 & 0.11 \\ O \times I \times T & 1,26 & 1.99 & 2.41 & 0.14 & 0.01 \\ & 1,26 & 1.63 & 0.95 & 1.66 & 0.01\end{array}$
a) $p<5 \%$ one-tailed.
b) $p<5 \%$ two-tailed.
c) $p<1 \%$ two-tailed. 

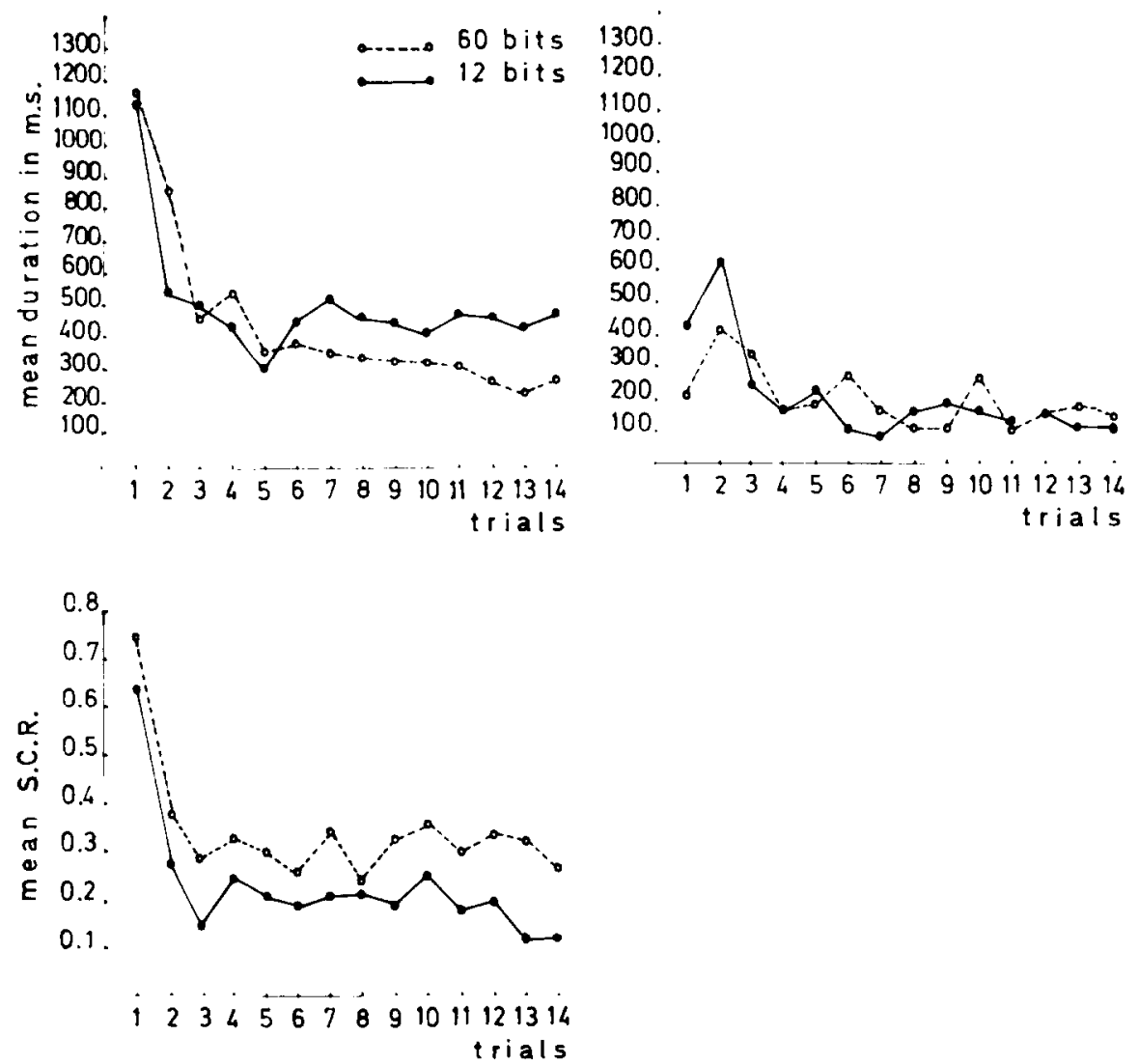

Fig. 2. Mean fixation durations inside field 1 and field 2 (above), and the mean SCR (below), during the two information conditions.

None of the interactions between the main factors was significant. This means that there was no indication for interactions between the two treatment phases, which might have led to asymmetrical effects in the balanced design used in this study (Poulton and Freeman, 1966).

SCR and VOR habituation scores were also analysed with a multivariate analysis of variance. Order of presentation of information conditions was a between-subjects factor and information a within-subjects factor. The results are summarized in table 2 (see also fig. 3).

The only significant effect was that of information on the SCR habituation scores; habituation scores were higher during the higher information condition.

In order to investigate the relationship between SCR habituation scores and SFs, we summated the SF scores for each subject over all 14 trials. Product-moment cor- 
Table 2

Summary of the multivariate analysis; univariate $F$ s and $d f$ for the VOR and SCR habituation scores

$\begin{array}{llll}\text { Source } & \text { df } & \text { VOR } & \text { SCR } \\ \text { Order }(O) & 1,26 & 0.37 & 0.98 \\ \text { Information }(I) & 1,26 & 0.09 & 8.45 \text { a) } \\ O \times I & 1,26 & 0.76 & 4.81 \text { b) }\end{array}$

a) $p<1 \%$ two-tailed.

b) $p<5 \%$ two-tailed.

relations (Ferguson, 1959) between SCR habituation scores and summated SF scores were highly significant during both information conditions (60-bits conditions: $[r=0.88(\mathrm{df}=26, p<1 \%$, two-tailed $)], 12$-bits condition $[r=0.68(\mathrm{df}=$ $26, p<1 \%$, two-tailed)].

In order to show that the changes of the VOR during the habituation trails were not caused by artefacts such as drift or head movements, fixations during trial 1 , during trial 14 and during the check procedure were scored for accuracy on an ordinal scale. An ordinal scale was used because the locus of eye fixation was the crucial variable and not fixation time. Score 2 was given for fixations inside field 1 , score 1 for fixations inside field 2 and score 0 for fixations outside these fields.

The results in table 3 indicate that there were no significant differences in locus of eye fixation between trial 1 and the check trial, but that these trials differed sig. nificantly from trial 14 (see table 3 and fig. 4).

The results of the recognition test were that $97 \%$ of the subjects correctly identified the 12-bits figure and $97 \%$ of the subjects the 60 -bits figure.

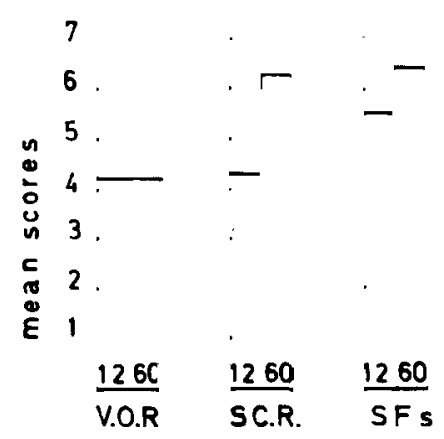

Fig. 3. Mean VOR and SCR habituation scores and the mean number of SFs during the two information conditions. 
Table 3

Results of the Wilcoxon matched pairs rank test; $z$-values and two-tailed $p$-values

Comparison Conditions

12 bits

60 bits

$z$

Trial $1 / 14$

z

Trial 14/check

$-3.80$

$-3.36$

$-0.67$

$p$

Trial $1 /$ check

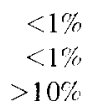

$-3.84$

$-3.87$

$-0.28$

$p$
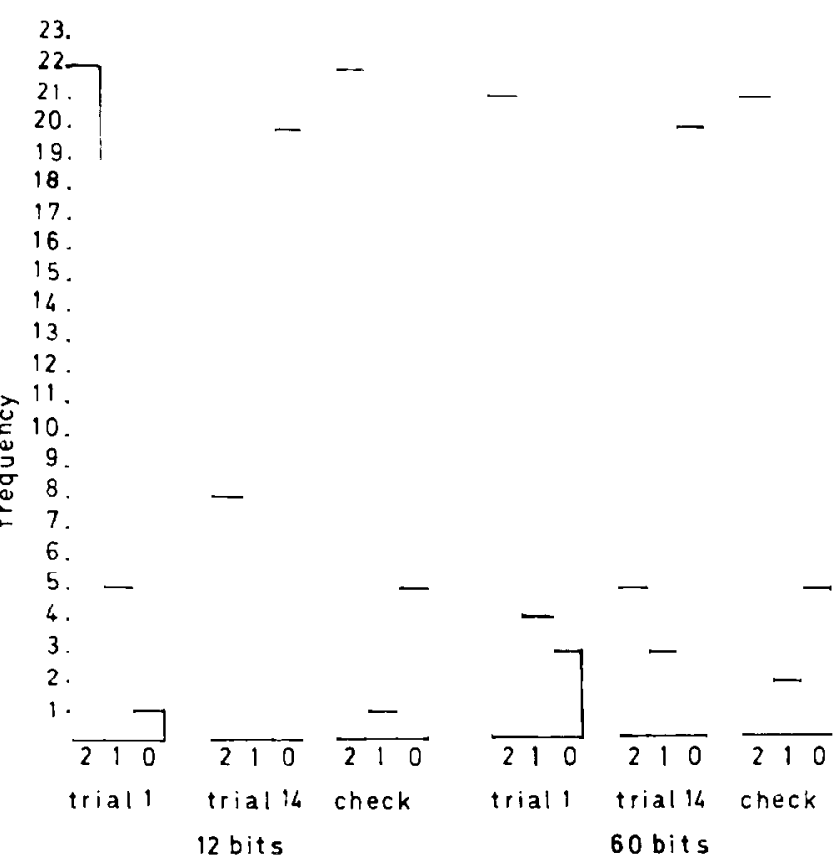

60 bits

Fig. 4. Number of subjects (frequency) which fixated during trial, trial 14 and the check trial inside field 1 , field 2 or outside these fields, in the 12 bits and the 60 bits condition.

\section{Discussion}

Because in section 1 the conclusion was drawn that task relevance might he a crucial variable when investigating the influence of the information value of stimuli on habituation rate of the $\mathrm{OR}$, it was essential to control whether our task-relevant 
condition had been effective. We therefore compared the performance on the recognition test in this study to that in a former study (Verbaten et al., 1979), in which the same figures were used, but no task-significance had been given to the stimuli. Recognition scores of the present study were both close to $100 \%$ correct. Comparison of these scores with these of the earlier study (Verbaten et al., 1979) showed that the task-relevance condition had been effective; the frequency of correct recognition test in this study to that in a former study (Verbaten et al., 1979), in which higher (Ch-square $=23.1, \mathrm{df}=1, p<1 \%$ ).

Of interest is the observation in this experiment that, although the two figures did not induce differences in the amount of time they were fixated, they induced different amounts of electrodermal activity; the subjects kept responding with the SCR to the 60 bits stimuli at the time that the VOR had already habituated. It could be argued that this lack of covariation between the two OR indices simply reflected the fact that, although the VOR and SCR measured the same thing, the SCR was more sensitive. In our opinion such an interpretation is contradicted by the results of a earlier study of the present authors (Verbaten et al., 1979) in which the VOR reflected the differences in information value of the stimuli, while the SCR did not.

Unexpectedly, stimulus information did not influence either the summated fixation time, nor VOR habituation rate. An explanation for these results might be that VOR habituation is among other things a function of the subject's uncertainty about the experimental situation. In this experiment subjects knew that only two different stimuli would be presented which they had to memorize. This in contrast to the more common instructions in habituation studies, in which the subjects are left uncertain about the stimulus situation. Research is in progress to investigate the influence of uncertainty on the VOR.

In accordance with the results of Spinks and Siddle (1976) we found slower SCR habituation to the stimuli with the higher information value. Unlike these authors we found, besides higher SCR habituation scores to the 60-bits figure, also larger SCR amplitudes. The most probable explanation is that the task in our study was more difficult than the task in the study of Spinks and Siddle (1976). Their subjects had to memorize only one figure, while the subjects in our study had to memorize two different figures, which might have given interference to some degree. It has been shown that more difficult tasks induce more electrodermal activity (Kahneman, 1969).

SFs did not habituate and were not influenced by the information value of the stimuli, but on the other hand correlated significantly with SCR habituation rate. This leads to the conclusion that SCR habituation is to some degree determined by autonomic arousal level, as indexed by number of SFs, but also reflects specific activity in relation to the processing of stimuli.

One explanation for the results of this study is that the VOR and the SCR reflect different stages of the information processing that takes place during the OR. There is some support in the literature for this suggestion. Probram and 
McGuinness (1975), discussing the dissociation between habituation of the galvanic skin response (GSR) and behavioral habituation of amygdalectomized monkeys, proposed that the GSR reflected not so much the behavioral orienting reaction, but the registration of the stimuli.

Cognitive psychologists (Reynolds and Flagg, 1977) assume that the information processing system consists of a sensory store (of unlimited capacity), an active or short term memory (of limited capacity) and a long term memory (of unlimited capacity). Just and Carpenter (1976) assume that eye movements reflect what is currently processed in the short term memory. But information in the STM tends to get lost unless the subject actively processes this information. Kahneman (1969) showed that active mental processing induced, besides pupil dilation, an increase in electrodermal activity.

The results of this study are interpreted against this background; the VOR might reflect a more primary information processing phase, while the SCR might reflect a more secondary phase, connected with 'registration' or active mental processing.

\section{References}

Attneave, F. (1954). Some informational aspects of visual perception. Psychological Review, 61, 183-193.

Berlyne, A.D., Craw, M.A., Salapatek, P.H. and Lewis, J.L. (1963). Novelty, complexity, incongruity, extrinsic motivation, and the GSR. Journal of Experimental Psychology, 66, 560-567.

Bernstein, A.S. (1973). Electrodermal lability and the OR: Reply to O'Gorman and further exposition of the significance hypothesis. Australian Journal of Psychology, 25, 147-154.

Carroll, D. and Pokora, J. (1976). The effects of threat of shock on SCR habituation to simple auditory stimuli. Physiological Psy chology, 4, 94-96.

Cook, S.W. and Harris, R.E. (1937). The verbal conditioning of the galvanic skin reflex. Journal of Experimental Psychology, 21, 202-210.

Ferguson. G.A. (1959). Statistical Analysis in Psychology and Education. McGra-Hill: New York, 136.

Finn, J.D. (1976). MULTIVARIANCE, Version V, Release 3. A Fortran IV Program. National Educational Resources Inc.: Chicago.

Grings, W.W. and Lockhart, R.A. (1963) Effects of 'anxiety-lessening' instructions and differential set development on the extinction of the GSR. Journal of Experimental Psychology, $66,292-299$.

Harding, G. and Punzo, l. (1971). Response uncertainty and skin conductance. Journal of Experimental Psychology, 88, 265-272.

Just, M.A. and Carpenter, P.A. (1976). Eye fixations and cognitive processes. Cognitive Psychology 8, 441-480.

Kahneman, D. (1969). Pupillary, heart rate and skin resistance changes during a mental task. Journal of Experimental Psychology, 79, 164-167.

Martin, I. and Rust, J. (1976). Habituation and the structure of the electrodermal system. Psychophysiology, 13, 554-562.

Poulton, E.C. and Freeman, P.R. (1960). Unwanted asymmetrical transfer effects with balanced designs, Psychological Bulletin, 66, 1-8. 
Pribram, K.H. and McGuinness, D. (1975). Arousal, attention, and effort in the control of attention. Psychological Review, 82(2), 116-149.

Reynolds, A.G. and Flagg, P.W. (1977). Cognitive Psychology. Winthrop Publishers Inc.: Mass., 218.

Siegel, S. (1956). Non-parametric Statistics for the Behavioral Sciences. McGraw-Hill: New York, $42-75$.

Sokolov, E.N. (1963). Perception and the Conditioned Retlex. Pergamon Press: Oxford.

Spinks, J.A. and Siddle, D.A.T. (1976). Effects of stimulus information and stimulus duration on amplitude and habituation of the electrodermal orienting response. Biological Psychology , 4, 29-39.

Verbaten, M.N., Woestenburg, J.C. and Sjouw, W. (1979). The influence of visual information on habituation of the electrodermal and the visual orienting reaction. Biological Psychology, $8,189-201$. 